\title{
Localization at the boundary for conditioned random walks in random environment in dimensions two and higher*
}

\author{
Rodrigo Bazaes ${ }^{\dagger}$
}

\begin{abstract}
We introduce the notion of localization at the boundary for conditioned random walks in i.i.d. and uniformly elliptic random environment on $\mathbb{Z}^{d}$, in dimensions two and higher. If $d=2$ or 3 , we prove localization for (almost) all walks. In contrast, for $d \geq 4$, there is a phase transition for environments of the form $\omega_{\varepsilon}(x, e)=\alpha(e)(1+\varepsilon \xi(x, e))$, where $\{\xi(x)\}_{x \in \mathbb{Z}^{d}}$ is an i.i.d. sequence of random variables, and $\varepsilon$ represents the amount of disorder with respect to a simple random walk.
\end{abstract}

Keywords: random walks; random environment; localization. MSC2020 subject classifications: 60K37; 82C41; 82D30.

Submitted to ECP on February 21, 2021, final version accepted on September 4, 2021. Supersedes arXiv: 1911.06430.

\section{Introduction}

In this paper, we deal with the notion of localization for random walks in random environment (RWRE). Informally, the walk is localized if its asymptotic trajectory is confined to some region with positive probability. Otherwise, it is delocalized. For RWRE, localization has been studied almost entirely in the one-dimensional case (see, for example, the works of Sinai [21] and Golosov [13]). This work aims to start the investigation of this phenomenon in dimensions greater than one, considering the case of the boundary. Let us first define the model on which we will be working.

\subsection{The model of a random walk in a random environment}

Fix $d \in \mathbb{N}$, the dimension where the walk moves. For $x \in \mathbb{R}^{d}$ and $p \in[1, \infty]$, the $\ell_{p}$ norm of $x$ is denoted by $|x|_{p}$. Define $V:=\left\{x \in \mathbb{Z}^{d}:|x|_{1}=1\right\}=\left\{ \pm e_{1}, \cdots, \pm e_{d}\right\}$ the set of allowed jumps of the walk (as usual, $e_{i}$ is the vector $(0, \cdots, \underbrace{1}_{\text {i-th position }}, \cdots 0)$ ). An environment is an element $\omega$ in the space $\Omega:=\mathcal{M}_{1}(V)^{\mathbb{Z}^{d}}$ (in general, we denote by $\mathcal{M}_{1}(X)$ to the space of probability measures on $X$ ). We usually write $\omega=\{\omega(x, e)\}_{x \in \mathbb{Z}^{d}, e \in V}$. Finally, we can define a random walk in the environment $\omega \in \Omega$ starting at a point $x \in \mathbb{Z}^{d}$ as the Markov chain $X=\left(X_{n}\right)_{n \in \mathbb{N}}$ with law $P_{x, \omega}$ that satisfies

${ }^{*}$ The author has been supported by ANID-PFCHA/Doctorado Nacional/ 2018-21180873.

${ }^{\dagger}$ Pontificia Universidad Católica de Chile. E-mail: rebazaes@mat.uc.cl 


$$
\begin{gathered}
P_{x, \omega}\left(X_{0}=x\right)=1, \\
P_{x, \omega}\left(X_{n+1}=y+e \mid X_{n}=y\right)= \begin{cases}\omega(y, e), & \text { if } P_{x, \omega}\left(X_{n}=y\right)>0 \\
0, & \text { otherwise. }\end{cases}
\end{gathered}
$$

The measure $P_{x, \omega}$ in the literature is known as the quenched measure, in contrast to the annealed (or averaged) measure we describe next. We use the following notation in the sequel: if $Y=\Omega$ or $Y=\left(\mathbb{Z}^{d}\right)^{\mathbb{N}}$, then $\mathbb{B}(Y)$ is its Borel $\sigma$-algebra. In our case, choose a probability measure $\mathbb{P}$ on $(\Omega, \mathbb{B}(\Omega))$. The annealed measure $P_{x}$ of the RWRE starting at $x \in \mathbb{Z}^{d}$ is defined as the measure on $\Omega \times\left(\mathbb{Z}^{d}\right)^{\mathbb{N}}$ that satisfies $P_{x}(A \times B)=\int_{A} P_{x, \omega}(B) d \mathbb{P}$ for each $A \in \mathbb{B}(\Omega)$ and $B \in \mathbb{B}\left(\left(\mathbb{Z}^{d}\right)^{\mathbb{N}}\right)$. Expectations with respect to $P_{x, \omega}, P_{x}$ and $\mathbb{P}$ are denoted by $E_{x, \omega}, E_{x}$ and $\mathbb{E}$ respectively. The basics assumptions in this work are the following:

\section{Assumption 1.1.}

(i) The random vectors $\{\omega(x, \cdot)\}_{x \in \mathbb{Z}^{d}}$ are i.i.d under $\mathbb{P}$.

(ii) Uniform ellipticity: there exists a $\kappa>0$ such that for every $x \in \mathbb{Z}^{d}$ and $e \in V$,

$$
\mathbb{P}(\omega(x, e) \geq \kappa)=1
$$

The two assumptions above are common in the literature. In particular, due to $(i)$, we can define $q(e):=\mathbb{E}[\omega(0, e)]=\mathbb{E}[\omega(x, e)]$ for $x \in \mathbb{Z}^{d}$ and $e \in V$.

More information about the model can be found in the references [12, 25].

\subsection{Localization at the boundary}

We will look at trajectories $\left(X_{n}\right)_{n \in \mathbb{N}}$ of an RWRE such that $\left|X_{n}\right|_{1}=n$ for each $n$, and study the asymptotic behavior of the normalized quenched probability of reaching the boundary at time $n$, that is, if $x \in \mathbb{Z}^{d}$ satisfies $|x|_{1}=n$,

$$
P_{0, \omega}\left(X_{n}=\left.x|| X_{n}\right|_{1}=n\right) .
$$

Specifically, we are concerned in knowing if for some sequence $\left(x_{n}\right)_{n \in \mathbb{N}} \subset \mathbb{Z}^{d}$ such that $|x|_{1}=n$ for all $n$, the quenched probability (1.3) is greater than some constant $c$, uniformly on $n$. In this case, the conditioned walk is "localized" around this path. There is a counterpart in the literature of directed polymers in random environment (see [8], p. 88). In this model, there is a nice characterization of localization/delocalization depending on the disorder of the environment. For RWRE, the disorder measures how far is the environment $\omega(0, e)$ from its expectation $q(e)$. This allows us to obtain analogous results in our case.

At this point, we proceed to characterize localization rigorously. We decompose $\partial \mathrm{D}$ in faces $\partial \mathbb{D}(s)$, with $s \in\{-1,1\}^{d}$, defined by $\partial \mathbb{D}(s):=\left\{x \in \partial \mathbb{D}: s_{j} x_{j} \geq 0, j=1, \cdots, d\right\}$. Set $\bar{s}:=(1,1, \cdots 1)$. From now on, we will restrict our attention to $\partial \mathbb{D}^{+}:=\partial \mathbb{D}(\bar{s})$; an analog treatment can be done on any face of the boundary. Define the allowed jumps by $V^{+}:=\left\{e_{1}, \cdots, e_{d}\right\} \subseteq V$. Next, consider the set

$$
\partial R_{n}:=n \partial \mathbb{D}^{+}=\left\{x \in \mathbb{Z}^{d}:|x|_{1}=n, x_{j} \geq 0 \text { for all } j \in\{1, \cdots, d\}\right\}
$$

and define $R_{n}$ as the sets of all paths $\left(z_{0}, z_{1}, \cdots, z_{n}\right) \in\left(\mathbb{Z}^{d}\right)^{n+1}$ for which $z_{0}=0$ and $z_{n} \in \partial R_{n}$. Note that this happens if and only if $\triangle z_{i}:=z_{i}-z_{i-1} \in V^{+}$for each $i=1, \cdots, n$. Subsequently, let $\mathcal{A}_{n}:=\left\{X_{n}-X_{0} \in \partial R_{n}\right\}$. Finally, the sequence of random variables $\left(J_{n}\right)_{n \in \mathbb{N}}$ is defined by $J_{1}:=1$, and for $n \geq 2$,

$$
J_{n}:=\max _{x \in \mathbb{Z}^{d}} P_{0, \omega}\left(X_{n-1}=x \mid \mathcal{A}_{n-1}\right) .
$$


Definition 1.2. An RWRE is localized at the boundary if

$$
\liminf _{n \rightarrow \infty} \frac{1}{n} \sum_{k=1}^{n} J_{k}>0 \mathbb{P}-\text { a.s. }
$$

and an RWRE is delocalized at the boundary if

$$
\liminf _{n \rightarrow \infty} \frac{1}{n} \sum_{k=1}^{n} J_{k}=0 \quad \mathbb{P}-a . s .
$$

Note that a priori, the walk can be neither localized nor delocalized. However, in Theorem 2.3 we show that this cannot happen for walks that satisfy Assumption 1.1.

\subsubsection{A different formulation}

Working on the boundary induces a polymer-like interpretation that makes more transparent the argument we use below. Given $\omega \in \Omega, x \in \mathbb{Z}^{d}$, and $e \in V^{+}$, define

$$
\pi(\omega, x, e):=\frac{\omega(x, e)}{\sum_{e^{\prime} \in V^{+}} \omega\left(x, e^{\prime}\right)}, \Psi(\omega, x):=\log \left(\sum_{e \in V^{+}} \omega(x, e)\right) .
$$

Then $\omega(x, e)=\pi(\omega, x, e) e^{\Psi(\omega, x)}$, and $\pi$ induces an RWRE, with $V^{+}$as the set of allowed jumps. Its quenched measure (starting at $x$ ) is $P_{x, \pi}$, and its expectation is $E_{x, \pi}$. Therefore, for fixed $n \in \mathbb{N}$ and $A \in \mathbb{B}\left(\left(\mathbb{Z}^{d}\right)^{\mathbb{N}}\right)$,

$$
P_{0, \omega}\left(A, X_{n} \in \partial R_{n}\right)=E_{0, \pi}\left(e^{\sum_{i=0}^{n-1} \Psi\left(\omega, X_{i}\right)}, A\right) .
$$

The last display leads to define a quenched polymer measure $P_{x, n}^{\omega}$ as

$$
P_{x, n}^{\omega}(A):=\frac{E_{0, \pi}\left(e^{\sum_{i=0}^{n-1} \Psi\left(\omega, X_{i}\right)}, A\right)}{E_{0, \pi}\left(e^{\sum_{i=0}^{n-1} \Psi\left(\omega, X_{i}\right)}\right)}, \quad A \in \mathbb{B}\left(\left(\mathbb{Z}^{d}\right)^{\mathbb{N}}\right) .
$$

This definition resembles the general framework introduced in [19]. Using the polymer measure, it is direct to verify the identity

$$
J_{n}=\max _{x \in \mathbb{Z}^{d}} P_{0, n-1}^{\omega}\left(X_{n-1}=x\right) .
$$

From now on, we use this scheme (except in Section 4.1), although, of course, both definitions are equivalent.

\subsection{Main results}

This paper's main results are that localization holds for (almost) all uniformly elliptic and i.i.d environments in dimensions two and three and a phase transition in terms of the disorder in dimensions four or higher.

Let $c:=\sum_{e \in V^{+}} q(e)$. The following assumption will play a remarkable role throughout the sequel.

Assumption 1.3. The measure $\mathbb{P}$ satisfies

$$
\mathbb{P}(\Psi(\omega, 0)=\log (c))<1 .
$$

Remark 1.4. As a consequence of Theorem 2.3, if Assumption 1.3 does not hold, then the walk is delocalized at the boundary for any $d \geq 2$. 
Theorem 1.5. Let $\left(X_{n}\right)_{n \in \mathbb{N}}$ be an RWRE that satisfies Assumptions 1.1 and 1.3. If $d \in\{2,3\}$, then the walk is localized at the boundary.

A related result in RWRE appears in the article [24] of Yilmaz and Zeitouni. They show that for walks that satisfy a certain ballisticity condition ${ }^{1}$, there is a class of measures $\mathbb{P}$ such that the quenched and annealed rate functions differ in a neighborhood of the LLN velocity. In the directed polymer model, Comets and Vargas [10] prove localization in dimension $1+1$ (one dimension for time, and one for space), while Lacoin [15] proves localization in dimension $1+2$. Berger and Lacoin improved this result in [5], where they gave the precise asymptotic behavior for the difference between the quenched and annealed free energies, as $n \rightarrow \infty$.

For $d \geq 4$, we consider a certain family of environments, parameterized by $\varepsilon \in[0,1)$. This parameter represents how much the distribution of the jumps in an RWRE differs from a simple random walk.

First, fix a probability vector $\alpha=(\alpha(e))_{e \in V}$ with strictly positive entries. Define

$$
\mathcal{E}_{\alpha}:=\left\{(r(e))_{e \in V} \in[-1,1]^{V}: \sum_{e \in V} r(e) \alpha(e)=0, \text { and } \sup _{e \in V}|r(e)|=1\right\}
$$

and consider a probability measure $\mathbb{Q}$ on $\Gamma_{\alpha}:=\mathcal{E}_{\alpha}^{Z^{d}}$ (also fixed from now). Next, pick an i.i.d family of random variables $(\xi(x))_{x \in \mathbb{Z}^{d}} \in \Gamma_{\alpha}$ such that $\mathbb{E}[\xi(x, e)]=0$ for all $e \in V$. Finally, given $\varepsilon \in[0,1)$, define the environments $\left(\omega_{\varepsilon}(x)\right)_{x \in \mathbb{Z}^{d}}$ as

$$
\omega_{\varepsilon}(x, e):=\alpha(e)(1+\varepsilon \xi(x, e)) .
$$

This framework was originally used in [4] to study a phase transition of the map $\varepsilon \mapsto$ $I_{a}(x, \cdot)-I_{q}(x, \cdot)$, where $I_{q}(x, \cdot), I_{a}(x, \cdot)$ are the quenched (respectively annealed) rate functions of an RWRE in the environment $\omega_{\varepsilon}$. The study of RWRE at low disorder has also been considered in $[20,22]$, among others.

For fixed $\varepsilon \in[0,1)$, let $\mathbb{P}_{\varepsilon}$ be the law of $\omega_{\varepsilon}$. This measure is uniformly elliptic with constant $\kappa=(1-\varepsilon) \min _{e \in V} \alpha(e)$. Conversely, for fixed $\kappa<\frac{1}{\min _{e \in V} \alpha(e)}$, we define $\varepsilon_{\max }:=1-\frac{\kappa}{\min _{e \in V} \alpha(e)}$, the maximum parameter so that for all $\varepsilon \leq \varepsilon_{\max }, \mathbb{P}_{\varepsilon}$ is uniformly elliptic with constant $\kappa$. The last result of the paper is the phase transition for localization/delocalization for parametrized environments. We say that an RWRE is $\varepsilon$-localized (resp. delocalized) if (1.5) (resp. (1.6)) holds under the measure $\mathbb{P}_{\varepsilon}$.

Theorem 1.6. For $d \geq 2, \alpha=(\alpha(e))_{e \in V}, \mathbb{Q}$ and $\kappa$ fixed, there exists $\bar{\varepsilon} \in\left[0, \varepsilon_{\max }\right]$ such that the walk is $\varepsilon$-localized for $0 \leq \varepsilon \leq \bar{\varepsilon}$, and $\varepsilon$-delocalized for $\bar{\varepsilon}<\varepsilon \leq \varepsilon_{\max }$. Moreover, if $d \geq 4$, then $\bar{\varepsilon}>0$. Also, there are examples of walks that satisfy $\bar{\varepsilon}<\varepsilon_{\max }$.

A direct consequence of Theorem 1.5 is the following:

Corollary 1.7. Under the same hypotheses and notation of Theorem 1.6, if Assumption 1.3 does not hold, then $\bar{\varepsilon}=\varepsilon_{\max }$. Otherwise, and if also $d=2$ or 3 , then $\bar{\varepsilon}=0$.

\subsubsection{Alternative approaches to localization}

In the recent article [3], the authors developed two notions related to localization in terms of the endpoint distribution $\rho_{n}(\cdot):=P_{0, n-1}^{\omega}\left(X_{n-1} \in \cdot\right)$ of a directed polymer in random environment.

(i) Given $i \in \mathbb{N}$ and $\varepsilon>0$, let $\mathcal{A}_{i}^{\varepsilon}:=\left\{x \in \mathbb{Z}^{d}: \rho_{i}(x) \geq \varepsilon\right\}$. We say $\left(\rho_{i}\right)_{i}$ is asymptotically purely atomic (concept introduced by Vargas in [23]) if, for each sequence $\left(\varepsilon_{i}\right)_{i}$

\footnotetext{
${ }^{1}$ Called condition (T). This condition is equivalent to the ballisticity conditions (T') and $\mathscr{P}_{M}$, as showed in [14]
} 
converging to zero as $i \rightarrow \infty$, it is true that

$$
\lim _{n \rightarrow \infty} \frac{1}{n} \sum_{i=1}^{n} \rho_{i}\left(\mathcal{A}_{i}^{\varepsilon_{i}}\right)=1 \mathrm{P}-\text { a.s. }
$$

(ii) Given $K, \delta>0$, let $\mathcal{G}_{\delta, K}$ be the set of probability distributions $\rho$ on $\mathbb{Z}^{d}$ for which there exists some set $A_{\delta, K} \subset \mathbb{Z}^{d}$ with diameter at most $K$, such that $\rho\left(\mathcal{A}_{\delta, K}\right) \geq 1-\delta$. We say that $\left(\rho_{i}\right)_{i}$ is geometrically localized with positive density if for each $\delta>0$, there exists some $K=K(\delta, d, \mathbb{P})$ and $\theta=\theta(\delta, d, \mathbb{P})>0$ such that

$$
\liminf _{n \rightarrow \infty} \frac{1}{n} \sum_{i=1}^{n} \mathbb{1}_{\left\{\rho_{i} \in \mathcal{G}_{\delta, K}\right\}} \geq \theta \mathbb{P}-\text { a.s. }
$$

For directed polymers, it is shown in the aforementioned article that if $\beta>\beta_{c}$ (i.e., the "low temperature" regime), then the endpoint distribution is both asymptotically purely atomic and geometrically localized with positive density. On the other hand, if $0 \leq \beta \leq \beta_{c}$ (i.e., the "high temperature" regime), then there is a sequence $\left(\varepsilon_{i}\right)_{i}$ that converges to zero as $i \rightarrow \infty$ such that

$$
\lim _{n \rightarrow \infty} \frac{1}{n} \sum_{i=1}^{n} \rho_{i}\left(\mathcal{A}_{i}^{\varepsilon_{i}}\right)=0 \mathbb{P}-a . s .
$$

Moreover, for any $K>0, \delta \in(0,1)$,

$$
\lim _{n \rightarrow \infty} \frac{1}{n} \sum_{i=1}^{n} \mathbb{1}_{\left\{\rho_{i} \in \mathcal{G}_{\delta, K}\right\}}=0 \mathrm{P}-\text { a.s. }
$$

Using the framework introduced in Section 1.2.1, the results above should be adaptable to our case for any $d \geq 2$ using the parametrization (1.12). On the other hand, in dimensions two and three, the endpoint distribution of an RWRE conditioned at the boundary should be both asymptotically purely atomic and geometrically localized with positive density (as soon as Assumption 1.3 is satisfied).

One of the novelties in [3] is a compactification argument for distributions on $\mathbb{Z}^{d}$, which was inspired by the analog treatment for distributions on $\mathbb{R}^{d}$ made in [17]. Further applications of similar ideas can be found in [2], [7], and [1].

\section{An equivalent criterion for localization}

In this section, we prove an equivalent criterion of localization/delocalization that will be used throughout the sequel. First, we need to define the following quantities.

Definition 2.1. Let $\left(X_{n}\right)_{n \in \mathbb{N}}$ be an RWRE. Define the limits

$$
\begin{aligned}
p(\omega) & :=\lim _{n \rightarrow \infty} \frac{1}{n} \log E_{0, \pi}\left(e^{\sum_{i=0}^{n-1} \Psi\left(\omega, X_{i}\right)}\right), \\
\lambda & :=\lim _{n \rightarrow \infty} \frac{1}{n} \log \mathbb{E} E_{0, \pi}\left(e^{\sum_{i=0}^{n-1} \Psi\left(\omega, X_{i}\right)}\right)=\log (c) .
\end{aligned}
$$

The last equality holds since the conditioned walk is directed.

In the directed polymer literature, these limits are known as quenched and annealed free energy, respectively. The existence of $p$ and the fact that it is deterministic hinge upon the following characterization in terms of $I_{q}$, the quenched rate function for RWRE (which is also deterministic, see Lemma 3.5 in [4]).

Lemma 2.2. For an RWRE that satisfies Assumption 1.1, the following identities hold:

$$
p=-\inf _{x \in \partial \mathbb{D}^{+}} I_{q}(x), \quad \lambda=-\inf _{x \in \partial \mathbb{D}^{+}} I_{a}(x) .
$$


The proof of this lemma follows the lines of Lemma 16.12 in [18], so it is omitted. Once the existence of $p$ and $\lambda$ is established, we proceed to state a criterion of localization/delocalization with regards to whether $p=\lambda$ or $p<\lambda$.

Theorem 2.3. Let $\left(X_{n}\right)_{n \in \mathbb{N}}$ be an RWRE that satisfies Assumption 1.1.

(i) The RWRE is localized at the boundary if and only if $p<\lambda$.

(ii) The RWRE is delocalized at the boundary if and only if $p=\lambda$.

In particular, the walk is either localized or delocalized $\mathrm{P}$-a.s.

As a corollary, we obtain a characterization of localization/delocalization in terms of the difference between the infima of the quenched and annealed rate functions.

Corollary 2.4. For an RWRE that satisfies Assumption 1.1, we have localization at the boundary if and only if

$$
\inf _{x \in \partial \mathbb{D}^{+}} I_{a}(x)<\inf _{x \in \partial \mathbb{D}^{+}} I_{q}(x) .
$$

To prove Theorem 2.3, we need to introduce a couple of definitions. The first is a martingale that relates $p$ and $\lambda$, and the second is a random variable linked to $J_{n}$.

Definition 2.5. Given an RWRE $\left(X_{n}\right)_{n \in \mathbb{N}}$ that satisfies Assumption 1.1, define the random variable in $(\Omega, \mathbb{B}(\Omega), \mathbb{P})$

$$
W_{n}(\omega):=E_{0, \pi}\left(e^{\sum_{i=0}^{n-1} \Psi\left(\omega, X_{i}\right)-n \log (c)}\right), \quad n \in \mathbb{N} .
$$

The following lemma is straightforward, so its proof is skipped.

Lemma 2.6. The process $\left\{W_{n}\right\}_{n \in \mathbb{N}}$ is a mean-one $\mathcal{F}_{n}$-martingale under the filtration $\left\{\mathcal{F}_{n}\right\}_{n \geq 0}$ given by $\mathcal{F}_{0}:=\{\emptyset, \Omega\}$, and for $n \geq 1, \mathcal{F}_{n}:=\left\{\omega(x, e):|x|_{1}<n, x \in \mathbb{Z}^{d}, e \in V^{+}\right\}$.

The martingale convergence theorem implies that $W_{\infty}:=\lim _{n \rightarrow \infty} W_{n}$ exists and is non-negative $\mathbb{P}$-a.s. Since the event $\left\{W_{\infty}=0\right\}$ is invariant under the translations $\left(T_{e}\right)_{e \in V^{+}} \mathbb{P}$-a.s., the ergodicity of $\mathbb{P}$ implies that $\mathbb{P}\left(W_{\infty}=0\right) \in\{0,1\}$. This consequence will be useful in Proposition 2.7.

Next, we introduce a second random variable,

$$
I_{n}(\omega):=\sum_{x \in \mathbb{Z}^{d}} P_{0, n-1}^{\omega}\left(X_{n-1}=x\right)^{2} .
$$

This random variable is $\mathcal{F}_{n-1}$-measurable. Observe that

$$
J_{n}^{2} \leq I_{n} \leq J_{n}
$$

The main ingredient in the proof of Theorem 2.3 is the next proposition, which compares $W_{n}$ and $I_{n}$. We use the following notation: for sequences $\left(a_{n}\right),\left(b_{n}\right)$ we say that $a_{n}=\Theta\left(b_{n}\right)$ if $a_{n}=O\left(b_{n}\right)$ and $b_{n}=O\left(a_{n}\right)$.

Proposition 2.7. Let $\left(X_{n}\right)_{n \in \mathbb{N}}$ be an RWRE that satisfies Assumption 1.1. Then the equality

$$
\left\{W_{\infty}=0\right\}=\left\{\sum_{n=1}^{\infty} I_{n}=\infty\right\}
$$

holds $\mathbb{P}$-a.s. Furthermore, if $\mathbb{P}\left(W_{\infty}=0\right)=1$, there exist constants $c_{1}(\mathbb{P}), c_{2}(\mathbb{P}) \in(0, \infty)$ for which $\mathbb{P}$-a.s.,

$$
c_{1} \sum_{k=1}^{n} I_{k} \leq-\log W_{n} \leq c_{2} \sum_{k=1}^{n} I_{k} \quad \text { for } n \text { large enough, }
$$


that is, $-\log W_{n}=\Theta\left(\sum_{k=1}^{n} I_{k}\right)$.

Sketch of the proof of Proposition 2.7. The proof of Theorem 2.1 in [9] can be adapted to show Proposition 2.7. It is based on the Doob's decomposition of the submartingale $-\log W_{n}$. More precisely, there exist a martingale $\left\{M_{n}\right\}_{n \in \mathbb{N}}$ and an adapted process $\left\{A_{n}\right\}_{n \in \mathbb{N}}$ such that for all $n \in \mathbb{N},-\log W_{n}=M_{n}+A_{n}$. Indeed, $A_{n}=-\sum_{i=1}^{n} \mathbb{E}\left[\log \left(\frac{W_{i}}{W_{i-1}}\right) \mid \mathcal{F}_{i-1}\right]$. Noting that

$$
\frac{W_{i}}{W_{i-1}}=E_{0, i-1}^{\omega}\left[e^{\Psi\left(\omega, X_{i-1}\right)-\log (c)}\right]=1+E_{0, i-1}^{\omega}\left[e^{\Psi\left(\omega, X_{i-1}\right)-\log (c)}-1\right]=: 1+U_{i}
$$

we decompose $A_{n}$ and $M_{n}$ as

$$
A_{n}=-\sum_{i=1}^{n} \mathbb{E}\left[\log \left(1+U_{i}\right) \mid \mathcal{F}_{i-1}\right], \quad M_{n}=\sum_{i=1}^{n}\left(-\log \left(1+U_{i}\right)+\mathbb{E}\left[\log \left(1+U_{i}\right) \mid \mathcal{F}_{i-1}\right]\right) .
$$

Exactly as in the aforementioned result, it is enough to prove that there is a constant $C>0$ such that for all $n \in \mathbb{N}$,

$$
\frac{1}{C} I_{n} \leq \mathbb{E}\left[-\log \left(1+U_{n}\right) \mid \mathcal{F}_{n-1}\right] \leq C I_{n}, \quad \mathbb{E}\left[\log ^{2}\left(1+U_{n}\right) \mid \mathcal{F}_{n-1}\right] \leq C I_{n}
$$

To check the inequalities above, notice that, by uniform ellipticity, the potential $\Psi$ is bounded $\mathbb{P}$-a.s., so there are constants $0<C_{1}<C_{2}$ such that $\mathbb{P}$-a.s., for all $n \in \mathbb{N}, \frac{W_{n}}{W_{n-1}} \in$ $\left(C_{1}, C_{2}\right)$. Therefore,

$$
U_{n}-C_{3} U_{n}^{2} \leq \log \left(1+U_{n}\right) \leq U_{n}-C_{4} U_{n}^{2}
$$

for some constants $C_{3}, C_{4}>0$. Thus, $\mathbb{E}\left[-\log \left(1+U_{n}\right) \mid \mathcal{F}_{n-1}\right]$ is bounded by above by

$$
\begin{aligned}
& \mathbb{E}\left[-U_{n}+C_{3} U_{n}^{2} \mid \mathcal{F}_{n-1}\right]=-C_{4} \mathbb{E}\left[U_{n}^{2} \mid \mathcal{F}_{n-1}\right] \\
& =C_{3} \sum_{x, x^{\prime} \in \mathbb{Z}^{d}} \mathbb{E}\left[E_{0, n-1}^{\omega}\left(e^{\Psi(\omega, x)-\log (c)}-1, X_{n-1}=x\right) \times\right. \\
& \left.E_{0, n-1}^{\omega}\left(e^{\Psi\left(\omega, x^{\prime}\right)-\log (c)}-1, X_{n-1}=x^{\prime}\right) \mid \mathcal{F}_{n-1}\right] \\
& =C_{3} \sum_{x, x^{\prime} \in \mathbb{Z}^{d}} \mathbb{E}\left[\left(e^{\Psi(\omega, x)-\log (c)}-1\right)\left(e^{\Psi\left(\omega, x^{\prime}\right)-\log (c)}-1\right)\right] \times \\
& P_{0, n-1}^{\omega}\left(X_{n-1}=x\right) P_{0, n-1}^{\omega}\left(X_{n-1}=x^{\prime}\right) \\
& =C_{3} \mathbb{E}\left[\left(e^{\Psi(\omega, 0)-\log (c)}-1\right)^{2}\right] I_{n} .
\end{aligned}
$$

Similarly we get a lower bound $\mathbb{E}\left[-\log \left(1+U_{n}\right) \mid \mathcal{F}_{n-1}\right] \geq C_{4} \mathbb{E}\left[\left(e^{\Psi(\omega, 0)-\log (c)}-1\right)^{2}\right] I_{n}$, and this shows the first inequality in (2.9). Finally, noting that for some constant $C_{5}>0$, $\log ^{2}\left(1+U_{n}\right) \leq C_{5} U_{n}^{2}$, repeating the steps from the last display we get the second inequality on (2.9), concluding the proof.

Proof of Theorem 2.3.

Recall that, due to (2.5), we have

$$
\left(\frac{1}{n} \sum_{k=1}^{n} J_{k}\right)^{2} \leq \frac{1}{n} \sum_{k=1}^{n} J_{k}^{2} \leq \frac{1}{n} \sum_{k=1}^{n} I_{k} \leq \frac{1}{n} \sum_{k=1}^{n} J_{k}
$$


Thus, the liminfs of the sequences $\left(\frac{1}{n} \sum_{k=1}^{n} I_{k}\right)_{n}$ and $\left(\frac{1}{n} \sum_{k=1}^{n} J_{k}\right)_{n}$ are of the same nature, that is, both are positive $\mathbb{P}$-a.s. or zero $\mathbb{P}$-a.s.

If $p<\lambda, W_{\infty}=0 \mathbb{P}$-a.s. To check this, observe that if $W_{\infty}>0$ then $\frac{\log W_{n}}{n} \rightarrow 0$, but at the same time $\frac{\log W_{n}}{n} \rightarrow p-\lambda=0$. So, if $p<\lambda$, then $W_{\infty}=0 \mathbb{P}$-a.s. By (2.6), $\sum_{n} I_{n}=\infty \mathbb{P}$ - a.s. and $-\log W_{n}=\Theta\left(\sum_{k=1}^{n} I_{k}\right)$. In particular, $\liminf _{n \rightarrow \infty} \frac{1}{n} \sum_{k=1}^{n} I_{n}>0$, so the RWRE is localized at the boundary.

Reciprocally, if the walk is localized, $\sum_{k=1}^{n} I_{k}=\infty$, so by (2.6), $-\log W_{n}=\Theta\left(\sum_{k=1}^{n} I_{k}\right)$, and then $-\frac{\log W_{n}}{n} \rightarrow p-\lambda>0$. This proves $(i)$, and the proof of $(i i)$ is analogous.

\section{Proof of Theorem 1.5}

\subsection{Preliminaries for the proof of Theorem 1.5}

The method of fractional moment and change of measure used in the proof was originally introduced by Derrida et al. in [11] for the pinning model. For directed polymers, Lacoin and Moreno used it for the first time in [16] on the hierarchical lattice, and Lacoin in [15] on $\mathbb{Z}^{d}$. Yilmaz and Zeitouni adapted the technique in [24] for random walks in random environment. As the proofs are similar, we only mention the main points of them and refer to the papers above for further details. More precisely, let $\phi(\theta):=\sum_{e \in V} q(e) e^{\langle\theta, z\rangle}$. In [24], the analog of showing that $p<\lambda$ in the space-time RWRE setting, is to demonstrate that for a sufficiently large set of points $\theta \in \mathbb{R}^{d}$,

$$
\lim _{n \rightarrow \infty} \frac{1}{n} \mathbb{E} \log E_{0, \omega}\left[e^{\left\langle\theta, X_{n}\right\rangle-n \log (\phi(\theta))}\right]<0,
$$

Comparing with

$$
p-\lambda=\lim _{n \rightarrow \infty} \frac{1}{n} \mathbb{E} \log \left[W_{n}\right]=\lim _{n \rightarrow \infty} \frac{1}{n} \mathbb{E} \log E_{0, \pi}\left(e^{\sum_{i=0}^{n-1} \Psi\left(\omega, X_{i}\right)-n \log (c)}\right),
$$

the main difference between the two models is that the potential $\Psi(\omega, x)$ is replaced by a tilt that depends on the steps of the walk, namely, $\Psi_{s t}(\theta, e):=e^{\langle\theta, e\rangle}$. This introduces a correlation that in our case is not present (see the paragraph below (3.9)). Thus, it is natural to apply the methods in [24] to deduce the desired result. We sketch the main ideas and differences in the next pages.

First, note that Theorem 2.3 implies immediately delocalization when (1.10) does not hold. Indeed, in this case, P-a.s $\Psi(\omega, x)=\log (c)$ for all $x \in \mathbb{Z}^{d}$, so by (2.1), $p=\log (c)=\lambda$. Hence, until the end of the proof we assume that (1.10) holds.

Let $\left\{\hat{X}_{n}\right\}_{n \in \mathbb{N}}$ be a simple random walk with jumps in $V^{+}$and law $\hat{P}$ that satisfies

$$
\hat{P}\left(\hat{X}_{n+1}=x+e \mid \hat{X}_{n}=x\right)=\frac{q(e)}{\sum_{e^{\prime} \in V^{+}} q\left(e^{\prime}\right)}, \quad x \in \partial R_{n}, e \in V^{+},
$$

and define $\mu:=\hat{E}\left(\hat{X}_{1}\right)$. Consider $N=n m$ with $n$ fixed (but large enough) and $m \rightarrow \infty$. Recall that $W_{N}(\omega)=E_{0, \pi}\left(e^{\sum_{i=0}^{N-1} \Psi\left(\omega, X_{i}\right)-N \log (c)}\right)$. We define, for $y \in \mathbb{Z}^{d}$,

$$
J_{y}:=\left(\left(y-\frac{1}{2}\right) \sqrt{n},\left(y+\frac{1}{2}\right) \sqrt{n}\right) \subset \mathbb{R}^{d} .
$$

Given $Y=\left(y_{1}, \cdots, y_{m}\right) \in\left(\mathbb{Z}^{d}\right)^{m}$, let

$$
W_{N}(\omega, Y):=E_{0, \pi}\left(e^{\sum_{i=0}^{N-1} \Psi\left(\omega, X_{i}\right)-N \log (c)}, X_{j n}-j n \mu \in J_{y_{j}}, \forall j \leq m\right)
$$

and decompose

$$
W_{N}(\omega)=\sum_{Y} W_{N}(\omega, Y) .
$$


The decomposition in (3.3) is well-founded, since $\mathbb{Z}^{d} \subset \bigcup_{y \in \Lambda} J_{y}$. By the inequality $\left(\sum_{i} a_{i}\right)^{1 / 2} \leq \sum_{i} a_{i}^{1 / 2}$, valid for countable indices, we obtain $\mathbb{E}\left[W_{N}(\omega)^{1 / 2}\right] \leq$ $\sum_{Y} \mathbb{E}\left[W_{N}(\omega, Y)^{1 / 2}\right]$. This inequality gives us

$p-\lambda=\lim _{N \rightarrow \infty} \frac{1}{N} \mathbb{E} \log \left[W_{N}\right] \leq \liminf _{N \rightarrow \infty} \frac{2}{N} \log \mathbb{E}\left[W_{N}^{1 / 2}\right] \leq \liminf _{N \rightarrow \infty} \frac{2}{N} \log \left(\sum_{Y} \mathbb{E}\left[W_{N}(\omega, Y)^{1 / 2}\right]\right)$.

Now, we estimate each expectation $\mathbb{E}\left[W_{N}(\omega, Y)\right]^{1 / 2}$, applying the change of measure. The plan is the following (recall that $N=m n$ with fixed $n$ ): fix $j \in\{1, \cdots, m\}, Y \in\left(\mathbb{Z}^{d}\right)^{m}$, and a square integer $n$. Also, $C_{1}$ is a constant to determine, and $y_{0}:=0$. Then define

$$
B_{j}:=\left\{(z, i) \in \mathbb{Z}^{d} \times \mathbb{N}:(j-1) n \leq i<j n,\left|z-i \mu-y_{j-1} \sqrt{n}\right| \leq C_{1} \sqrt{n}\right\} .
$$

\subsection{Proof in the case $d=2$}

The idea is to define a function that depends on the different blocks $B_{j}$. Let

$$
\tilde{\omega}(y):=e^{\Psi(\omega, y)}-c, \text { and } \quad D\left(B_{j}\right):=\sum_{y:\left(y,|y|_{1}\right) \in B_{j}} \tilde{\omega}(y) .
$$

In particular, $\mathbb{E}\left[D\left(B_{j}\right)\right]=0$, and they form an independent family of random variables. It is important to observe that (1.10) guarantees that $\tilde{\omega}$ and $D\left(B_{j}\right)$ are non-degenerate random variables. We also define $\delta_{n}:=C_{1}^{-1 / 2} n^{-3 / 4}$. Note that $\delta_{n}^{2}\left|D\left(B_{1}\right)\right|=O(1)$. Finally, for $K>0$ large enough (to determine), define

$$
f_{K}(u):=-K \mathbb{1}_{\left\{u \geq e^{K^{2}}\right\}}, \quad g(\omega, Y):=e^{\sum_{j=1}^{m} f_{K}\left(\delta_{n} D\left(B_{j}\right)\right)} .
$$

By Cauchy-Schwarz inequality,

$$
\begin{aligned}
& \mathbb{E}\left[W_{N}(\omega, Y)^{1 / 2}\right]=\mathbb{E}\left[W_{N}(\omega, Y)^{1 / 2} g(\omega, Y)^{1 / 2} g(\omega, Y)^{-1 / 2}\right] \\
& \leq \mathbb{E}\left[W_{N}(\omega, Y) g(\omega, Y)\right]^{1 / 2} \mathbb{E}\left[g(\omega, Y)^{-1}\right]^{1 / 2} .
\end{aligned}
$$

One can show that for $K$ large enough, $\mathbb{E}\left[g(\omega, Y)^{-1}\right]^{1 / 2} \leq 2^{m}$. To bound $\mathbb{E}\left[W_{N}(\omega, Y) g(\omega, Y)\right]$, we can the estimates in Pages 251-252 from [24] to deduce that

$\mathbb{E}\left[W_{N}(\omega, Y)^{1 / 2}\right] \leq\left(2 \sum_{y \in \mathbb{Z}^{2}} \max _{x \in J_{0}} \mathbb{E} E_{x, \pi}\left(e^{\sum_{i=0}^{n-1} \Psi\left(\omega, X_{i}\right)+f_{K}\left(\delta_{n} D\left(B_{1}\right)\right)-n \log (c)} ; X_{n}-n \mu \in J_{y}\right)\right)^{m}$.

The bound (3.4) tell us that $p-\lambda<0$ once we are able to prove the following:

Lemma 3.1. For $n, K$, and $C_{1}$ large enough,

$$
\sum_{y \in \mathbb{Z}^{2}} \max _{x \in J_{0}} \mathbb{E} E_{x, \pi}\left(e^{\sum_{i=0}^{n-1} \Psi\left(\omega, X_{i}\right)+f_{K}\left(\delta_{n} D\left(B_{1}\right)\right)-n \log (c)} ; X_{n}-n \mu \in J_{y}\right)<1 / 2 .
$$

The proof of the lemma above is followed almost exactly from Section 2.5 in [24]. The main difference rests in display (2.22) in the aforementioned paper. In our case, we need to check that for some $\alpha>0$,

$$
\mathbb{E} E_{0, \pi}\left[e^{\sum_{i=0}^{n-1} \Psi\left(\omega, X_{i}\right)-n \log (c)}\left(\sum_{i=0}^{n-1} \tilde{\omega}\left(X_{i}\right)-\alpha\right)^{2}\right]
$$


is $O(n)$.

We can decompose it as

$$
\begin{aligned}
& \sum_{j=1}^{n-1} \mathbb{E} E_{0, \pi}\left[e^{\sum_{i=0}^{n-1} \Psi\left(\omega, X_{i}\right)-n \log (c)}\left(\tilde{\omega}\left(X_{j}\right)-\alpha\right)^{2}\right]+ \\
& 2 \sum_{1 \leq \ell<j \leq n-1} \mathbb{E} E_{0, \pi}\left[e^{\sum_{i=0}^{n-1} \Psi\left(\omega, X_{i}\right)-n \log (c)}\left(\tilde{\omega}\left(X_{\ell}\right)-\alpha\right)\left(\tilde{\omega}\left(X_{j}\right)-\alpha\right)\right] .
\end{aligned}
$$

The first term is $n \mathbb{E} E_{0, \pi}\left[e^{\Psi\left(\omega, X_{1}\right)-\log (c)}\left(\tilde{\omega}\left(X_{1}\right)-\alpha\right)^{2}\right]$. As $c_{n}:=\frac{\delta_{n}^{2}}{\left(\mu n \delta_{n}-A_{n}-e^{K^{2}}\right)^{2}}=O\left(n^{-2}\right)$, this expression vanishes as $n \rightarrow \infty$. On the other hand, if we choose

$$
\alpha:=\mathbb{E} E_{0, \pi}\left[e^{\Psi\left(\omega, X_{1}\right)-\log (c)} \tilde{\omega}\left(X_{1}\right)\right]=\frac{\mathbb{E}\left[e^{2 \Psi(\omega, 0)}\right]-c^{2}}{c}>0(\text { by (1.10)), }
$$

then by independence, the second term in (3.8) is zero. By comparison, the analog of $\alpha$ (called $\mu$ in [24]) is greater than zero due to a positive correlation that in our case is not needed.

Combining the previous results, such election of constants help us to deduce that Lemma 3.1 is true, and therefore $p-\lambda<0$.

\subsection{Proof in case $d=3$}

In this case, the proof in spirit is essentially the same, but some technical details need to be adapted to this situation. In particular, we need to redefine $\delta_{n}$ and $D\left(B_{j}\right)$. First, for a constant $C_{2}>0$ to determine, let

$$
V(y, z):=\frac{1}{|i-j|} \mathbb{1}_{\left\{|y-z-(i-j) \mu|<C_{2} \sqrt{|i-j|\}}\right.} \text { if } i \neq j, \text { and } 0 \text { otherwise. }
$$

Also, recall that $\tilde{\omega}$ is defined as in (3.6). Then we redefine

$$
\delta_{n}:=n^{-1}(\log n)^{-1 / 2}, \quad D\left(B_{j}\right):=\sum_{\substack{y, z \\(y, i),(z, j) \in B j}} V(y, z) \tilde{\omega}(y) \tilde{\omega}(z) .
$$

The proof of Theorem 1.6 in [24] can be followed almost word by word, and our situation is a little bit simplified since the correlation issue is not present as in the $d=2$ case. Details are omitted.

\section{Phase transition}

Recall the parametrization of the environments $\left(\omega_{\varepsilon}\right)_{\varepsilon \in[0,1)}$ (i.e., (1.12)). Let be $p(\varepsilon)$ the limit in (2.1) with environment $\omega_{\varepsilon}$. On the other hand, $\lambda$ is constant over $\varepsilon$, and it is equal to $\log \left(\sum_{e \in V^{+}} \alpha(e)\right)$. The first part of Theorem 1.6 is consequence of the lemma below:

Lemma 4.1. For each $n \in \mathbb{N}$, the map

$$
\varepsilon \in\left[0, \varepsilon_{\max }\right] \rightarrow \frac{1}{n}\left[\mathbb{E} \log P_{0, \omega_{\varepsilon}}\left(X_{n} \in \partial R_{n}\right)-\log P_{0}\left(X_{n} \in \partial R_{n}\right)\right] \text { is non-increasing. }
$$

This is an adaptation of Lemma 5.1 in [4]. If we let $n$ to infinity, then we deduce that $p(\varepsilon)-\lambda$ is non-increasing. To finish the proof, define

$$
\bar{\varepsilon}:=\inf \left\{\varepsilon \in\left(0, \varepsilon_{\max }\right]: p(\varepsilon)-\lambda<0\right\},
$$

with the convention that $\inf \emptyset=\varepsilon_{\max }$. 
The rest of this section is devoted to prove the second part of Theorem 1.6. The main ingredient to show that $\bar{\varepsilon}>0$ is the next lemma, a particular case of Lemma 3.1 with $\theta=0$ in [4].

Lemma 4.2. If $\varepsilon>0$ is small enough, then $\sup _{n}\left|W_{n}^{2}\right|_{2}<\infty$.

Recall the following: if $W_{\infty}(\varepsilon):=W_{\infty}\left(\omega_{\varepsilon}\right)$, then $W_{\infty}\left(\omega_{\varepsilon}\right)>0$ implies $p(\varepsilon)=\lambda$, the later being equivalent to localization. Indeed, If $W_{\infty}>0$, then $\log \left(W_{\infty}\right)=\lim _{n \rightarrow \infty} \log \left(W_{n}\right)<$ $\infty$, so

$$
p(\varepsilon)=\lim _{n \rightarrow \infty} \frac{1}{n} \log P_{0, \omega_{\varepsilon}}\left(X_{n} \in \partial R_{n}\right)=\lim _{n \rightarrow \infty} \frac{W_{n}\left(\omega_{\varepsilon}\right)}{n}+\lambda=\lambda .
$$

Now pick $\varepsilon>0$ small enough such that $\sup _{n}\left|W_{n}^{2}\right|_{2}<\infty$ as in Lemma 4.2, and call it $\varepsilon^{*}$. By the martingale convergence theorem, $W_{n}\left(\varepsilon^{*}\right) \rightarrow W_{\infty}\left(\varepsilon^{*}\right)$ a.s. and in $L^{2}$. As $\left|W_{n}\right|_{2}=1$ for all $n$, then we necessarily have $W_{\infty}\left(\varepsilon^{*}\right)>0$, and therefore $p\left(\varepsilon^{*}\right)=\lambda$. But the map $\varepsilon \rightarrow p(\varepsilon)-\lambda$ is non-increasing, so $p=\lambda$ on $\left[0, \varepsilon^{*}\right]$, and thus $\bar{\varepsilon} \geq \varepsilon^{*}>0$.

It only remains to show an example in dimension greater or equal than 4 where $0<\bar{\varepsilon}<\varepsilon_{\max }$.

\subsection{An example on which $\bar{\varepsilon}<\varepsilon_{\max }$}

For simplicity, we consider $d=4$, and i.i.d random variables $(\xi(x))_{x \in \mathbb{Z}^{d}} \in \Gamma_{\alpha}$ such that $\xi(x, e)=\xi\left(x, e^{\prime}\right)$ for all $e, e^{\prime} \in V^{+}$, and $\xi(x,-e)=-\xi(x, e)$. If $y=\left(y_{1}, \cdots, y_{d}\right) \in \partial \mathbb{D}^{+}$ is a point to determine, for $i=1, \cdots, d$, define $\alpha\left(e_{i}\right)=\alpha\left(-e_{i}\right):=\frac{y_{i}}{2 \sum_{i=1}^{d} y_{i}}$. Recall that

$$
\omega_{\varepsilon}\left(x, e_{i}\right)=\alpha\left(e_{i}\right)(1+\varepsilon \xi(x)) \quad e_{i} \in V^{+} .
$$

Moreover, assume that the distribution of $\xi(0)$ under $\mathbb{Q}$ is the Rademacher distribution, namely, $\mathbb{Q}(\xi(0)=1)=\mathbb{Q}(\xi(0)=-1)=\frac{1}{2}$. By Corollary 2.4, localization occurs as soon as $\inf _{x \in \partial \mathbb{D}^{+}} I_{a}(x)<\inf _{x \in \partial \mathbb{D}^{+}} I_{q}(x)$. However, in this case, the infimum on the left is exactly $I_{a}(y)$, and it is achieved only at this point (see Theorem 2.3 in [4]). On the other hand, by the continuity of $I_{q}$, the infimum on the right is also achieved at some point $\bar{x} \in \partial \mathbb{D}^{+}$. If $\bar{x} \neq y$, then $I_{a}(y)<I_{a}(\bar{x}) \leq I_{q}(\bar{x})$, so we are done. Thus, assume that $\bar{x}=y$. Denote by $\left(y_{n}\right)_{n \in \mathbb{N}}$ any sequence as in Lemma 3.5 in [4] for the point $y$. Then we decompose $-I_{q}(y)$ as

$$
\begin{aligned}
-I_{q}(y) & =-I_{a}(y)+\lim _{n \rightarrow \infty} \frac{1}{n} \log \left(\frac{\sum_{0=x_{0}, x_{1}, \cdots, x_{n}=y_{n}} \prod_{i=1}^{n} q\left(\triangle x_{i}\right)\left(1+\varepsilon \xi\left(x_{i-1}\right)\right)}{\sum_{0=x_{0}, x_{1}, \cdots, x_{n}=y_{n}} \prod_{i=1}^{n} q\left(\triangle x_{i}\right)}\right) \\
& \leq-I_{a}(y)+\limsup _{n \rightarrow \infty} \max _{0=x_{0}, x_{1}, \cdots, y_{n}} \frac{1}{n} \sum_{i=1}^{n} \log \left(1+\varepsilon \xi\left(x_{i-1}\right)\right) .
\end{aligned}
$$

Also, the sum and maximum above are over all directed paths $0=x_{0}, x_{1}, \cdots, x_{n}$ such that $x_{n}=y_{n}$. Let $C\left(y_{n}\right)$ be the number of such paths. It's easy to check that there exists some constant $C>0$ such that, for all $n \in \mathbb{N}, C\left(y_{n}\right) \leq C e^{n f(y)-\frac{d-1}{2} \log n}$, where $f(y)=$ $-\sum_{i=1}^{d} y_{i} \log \left(y_{i}\right)$. To estimate the maximum above, we can use Hoeffding inequality (see Theorem 2.8 in [6]) to obtain, for $a>0$,

$$
\mathbb{P}\left(\sum_{i=1}^{n} \log \left(1+\varepsilon \xi\left(x_{i-1}\right)\right)-n \mathbb{E}[\log (1+\varepsilon \xi(0))]>n a\right) \leq \exp \left(\frac{-2 n a^{2}}{\log \left(\frac{1+\varepsilon}{1-\varepsilon}\right)^{2}}\right)
$$


Therefore,

$$
\begin{aligned}
& \sum_{n=1}^{\infty} \mathbb{P}\left(\underset{0=x_{0}, x_{1}, \cdots, y_{n}}{\left.\max _{i=1}^{n} \log \left(1+\varepsilon \xi\left(x_{i-1}\right)\right)-n \mathbb{E}[\log (1+\varepsilon \xi(0))]>n a\right)}\right. \\
& \leq \sum_{n=1}^{\infty} C\left(y_{n}\right) \exp \left(\frac{-2 n a^{2}}{\log \left(\frac{1+\varepsilon}{1-\varepsilon}\right)^{2}}\right)<\infty
\end{aligned}
$$

as soon as $a>\log \left(\frac{1+\varepsilon}{1-\varepsilon}\right) \sqrt{f(y) / 2}$. By Borel-Cantelli's lemma, (4.1) is bounded by

$$
\begin{aligned}
& -I_{a}(y)+\log \left(\frac{1+\varepsilon}{1-\varepsilon}\right) \sqrt{f(y) / 2}+\mathbb{E}[\log (1+\varepsilon \xi(0))] \\
& =-I_{a}(y)+\log \left(\frac{1+\varepsilon}{1-\varepsilon}\right) \sqrt{f(y) / 2}+\frac{1}{2}(\log (1+\varepsilon)+\log (1-\varepsilon)) .
\end{aligned}
$$

If $f(y) \leq \frac{9}{50}$, then $\sqrt{f(y) / 2} \leq \frac{3}{10}$, and the last display is strictly smaller than $-I_{a}(y)$ at least for $\varepsilon>\frac{9}{10}$. The required value for $f(y)$ can be achieved, for example, selecting the vector $y=\left(\frac{97}{100}, \frac{1}{100}, \frac{1}{100}, \frac{1}{100}\right)$, so in this case, we can choose $\varepsilon_{\max } \approx \frac{9}{10}$ to obtain a true phase transition, with $\kappa \approx \frac{1}{1000}$.

Remark 4.3. The asymmetry in terms of $\alpha$ is needed. Indeed, if $\alpha(e)=\frac{1}{2 d}$ for all $e \in V$, then it is not difficult to show (see pp. 36-37 in [8]) that under our setting, $\sup _{n \in \mathbb{N}} \mathbb{E}\left[W_{n}^{2}\right]<\infty$, and therefore, $p(\varepsilon)=\lambda$ for all $\varepsilon \in\left[0, \varepsilon_{\max }\right]$.

\section{References}

[1] Yuri Bakhtin and Donghyun Seo, Localization of directed polymers in continuous space, Electron. J. Probab. 25 (2020), Paper No. 142, 56. MR-4186261

[2] Erik Bates, Localization of directed polymers with general reference walk, Electron. J. Probab. 23 (2018), Paper No. 30, 45. MR-3785400

[3] Erik Bates and Sourav Chatterjee, The endpoint distribution of directed polymers, Ann. Probab. 48 (2020), no. 2, 817-871. MR-4089496

[4] Rodrigo Bazaes, Chiranjib Mukherjee, Alejandro F. Ramírez, and Santiago Saglietti, The effect of disorder on quenched and averaged large deviations for random walks in random environment: boundary behavior, arXiv preprint arXiv:2101.04606 (2021).

[5] Quentin Berger and Hubert Lacoin, The high-temperature behavior for the directed polymer in dimension $1+2$, Ann. Inst. Henri Poincaré Probab. Stat. 53 (2017), no. 1, 430-450. MR-3606747

[6] Stéphane Boucheron, Gábor Lugosi, and Pascal Massart, Concentration inequalities, Oxford University Press, Oxford, 2013, A nonasymptotic theory of independence, With a foreword by Michel Ledoux. MR-3185193

[7] Yannic Bröker and Chiranjib Mukherjee, Localization of the Gaussian multiplicative chaos in the Wiener space and the stochastic heat equation in strong disorder, Ann. Appl. Probab. 29 (2019), no. 6, 3745-3785. MR-4047991

[8] Francis Comets, Directed polymers in random environments, Lecture Notes in Mathematics, vol. 2175, Springer, Cham, 2017, Lecture notes from the 46th Probability Summer School held in Saint-Flour, 2016. MR-3444835

[9] Francis Comets, Tokuzo Shiga, and Nobuo Yoshida, Directed polymers in a random environment: path localization and strong disorder, Bernoulli 9 (2003), no. 4, 705-723. MR-1996276

[10] Francis Comets and Vincent Vargas, Majorizing multiplicative cascades for directed polymers in random media, ALEA Lat. Am. J. Probab. Math. Stat. 2 (2006), 267-277. MR-2249671 
[11] Bernard Derrida, Giambattista Giacomin, Hubert Lacoin, and Fabio Lucio Toninelli, Fractional moment bounds and disorder relevance for pinning models, Comm. Math. Phys. 287 (2009), no. 3, 867-887. MR-2486665

[12] Alexander Drewitz and Alejandro F. Ramírez, Selected topics in random walks in random environment, Topics in percolative and disordered systems, Springer Proc. Math. Stat., vol. 69, Springer, New York, 2014, pp. 23-83. MR-3229286

[13] A. O. Golosov, Localization of random walks in one-dimensional random environments, Comm. Math. Phys. 92 (1984), no. 4, 491-506. MR-736407

[14] Enrique Guerra and Alejandro F. Ramírez, A proof of Sznitman's conjecture about ballistic RWRE, Comm. Pure Appl. Math. 73 (2020), no. 10, 2087-2103.

[15] Hubert Lacoin, New bounds for the free energy of directed polymers in dimension $1+1$ and $1+2$, Comm. Math. Phys. 294 (2010), no. 2, 471-503. MR-2579463

[16] Hubert Lacoin and Gregorio Moreno, Directed polymers on hierarchical lattices with site disorder, Stochastic Process. Appl. 120 (2010), no. 4, 467-493. MR-2594367

[17] Chiranjib Mukherjee and S. R. S. Varadhan, Brownian occupation measures, compactness and large deviations, Ann. Probab. 44 (2016), no. 6, 3934-3964. MR-3572328

[18] Firas Rassoul-Agha and Timo Seppäläinen, A course on large deviations with an introduction to Gibbs measures, Graduate Studies in Mathematics, vol. 162, American Mathematical Society, Providence, RI, 2015. MR-3309619

[19] Firas Rassoul-Agha, Timo Seppäläinen, and Atilla Yilmaz, Quenched free energy and large deviations for random walks in random potentials, Comm. Pure Appl. Math. 66 (2013), no. 2, 202-244. MR-2999296

[20] Christophe Sabot, Ballistic random walks in random environment at low disorder, Ann. Probab. 32 (2004), no. 4, 2996-3023. MR-2094437

[21] Ya. G. Sinal, The limit behavior of a one-dimensional random walk in a random environment, Teor. Veroyatnost. i Primenen. 27 (1982), no. 2, 247-258. MR-657919

[22] Alain-Sol Sznitman, On new examples of ballistic random walks in random environment, Ann. Probab. 31 (2003), no. 1, 285-322. MR-1959794

[23] Vincent Vargas, Strong localization and macroscopic atoms for directed polymers, Probab. Theory Related Fields 138 (2007), no. 3-4, 391-410. MR-2299713

[24] Atilla Yilmaz and Ofer Zeitouni, Differing averaged and quenched large deviations for random walks in random environments in dimensions two and three, Comm. Math. Phys. 300 (2010), no. 1, 243-271. MR-2725188

[25] Ofer Zeitouni, Random walks in random environment, Lectures on probability theory and statistics, Lecture Notes in Math., vol. 1837, Springer, Berlin, 2004, pp. 189-312. MR2071631

Acknowledgments. The author thanks his advisor Alejandro F. Ramírez and an anonymous referee for valuable comments and suggestions about the paper. Also, the author is grateful to Gregorio Moreno for a couple of discussions and bibliographical remarks. 


\section{Electronic Journal of Probability Electronic Communications in Probability}

\section{Advantages of publishing in EJP-ECP}

- Very high standards

- Free for authors, free for readers

- Quick publication (no backlog)

- Secure publication $\left(\mathrm{LOCKSS}^{1}\right)$

- Easy interface (EJMS²)

\section{Economical model of EJP-ECP}

- Non profit, sponsored by $\mathrm{IMS}^{3}, \mathrm{BS}^{4}$, ProjectEuclid ${ }^{5}$

- Purely electronic

\section{Help keep the journal free and vigorous}

- Donate to the IMS open access fund ${ }^{6}$ (click here to donate!)

- Submit your best articles to EJP-ECP

- Choose EJP-ECP over for-profit journals

\footnotetext{
${ }^{1}$ LOCKSS: Lots of Copies Keep Stuff Safe http://www. lockss.org/

${ }^{2}$ EJMS: Electronic Journal Management System http://www.vtex.lt/en/ejms.html

${ }^{3}$ IMS: Institute of Mathematical Statistics http://www.imstat.org/

${ }^{4}$ BS: Bernoulli Society http://www. bernoulli-society.org/

${ }^{5}$ Project Euclid: https://projecteuclid.org/

${ }^{6}$ IMS Open Access Fund: http://www.imstat.org/publications/open.htm
} 